\title{
Ideomotor apraxia and cerebral dominance for motor control
}

\author{
Georg Goldenberg ${ }^{\mathrm{a}, \mathrm{b}, *}$, Joachim Hermsdörfer ${ }^{\mathrm{b}}$, Joseph Spatt ${ }^{\mathrm{a}}$ \\ a 2nd Neurological Department of Neurological Hospital Rosenhügel, Riedelgasse 5, A 1130 Wien, Austria \\ ${ }^{\mathrm{b}}$ Neuropsychological Department of the City Hospital Bogenhausen, Englschalkingerstr. 77, D 81925 München, Germany
}

Accepted 22 August 1995

\begin{abstract}
Ideomotor apraxia is a symptom of left hemisphere damage. Patients with ideomotor apraxia commit errors when imitating movements with their left, non-paralyzed hand. This has been taken as evidence for a motor dominance of the left hemisphere. It has been hypothesized that the left hemisphere is dominant for internal preprogramming of skilled movements of either hand. We investigated the kinematics of movement trajectories of imitation of meaningless gestures. Group analysis confirmed that hesitant, feedback-controlled movement prevail in patients with apraxia, but analysis of single cases revealed the existence of kinematically normal movements leading to apractic errors. Enhanced reliance on feedback-control appears to be a compensatory strategy rather than the source of apractic errors. In a second study we explored the alternative hypothesis that patients with apraxia lack a general concept of the human body which is necessary to mediate the translation of a target position seen on the model into a target position on the patient's body. Imitation of movements was examined on oneself and on a mannikin. Patients with apraxia who made errors when imitating on themselves committed errors also when imitating on the mannikin. Taken together, both studies support the view that the source of errors in the imitation of gestures is to be sought at a conceptual level. This casts doubts on the alleged dominance of the left hemisphere for motor control.
\end{abstract}

Keywords: Apraxia; Motor control; Kinematics; Body-schema; Cerebral dominance; Neuropsychology

\section{Introduction}

Limb apraxia is a symptom of left hemisphere damage which is easily to demonstrate but difficult to understand. Apractic patients commit spatial and temporal errors when performing movements with their left, non-paretic hand. Errors may concern the use of objects and tools as well as movements performed without external objects like the pantomime of object use or the demonstration of conventional symbolic gestures. Apractic patients may commit errors even when the correct movements are demonstrated to them and they only have to imitate them [16].

The riddle of apraxia is posed by the observation that apractic patients can use their left hand normally in situations which are different from those of apraxia testing. The widely accepted definition of apraxia as a 'disorder of skilled movement not caused by weakness, akinesia, deafferentation, abnormal tone or posture, movement disorders

\footnotetext{
* Corresponding author. Neurological Department, Krankenhaus Bogenhausen, Englschalkingerstrasse 77, D-81925 München, Germany. Fax: (49) (89) $9270-2089$.
}

(such as tremor or chorea)..' [6] implies that there are situations where the left hand is moved with completely normal skill and velocity. Otherwise, the exclusion of other movement disorders could not be verified.

An explanation for the dissociation between normal and abnormal motor control of the left hand could be sought in different conceptual demands of the motor tasks examined. Such an explanation can easily account for errors occurring with tool use, pantomime of object use and symbolic gestures, as in all of them a correct concept of the intended movement has to be evoked from memory before movement execution can start. It does, however, have problems with impaired imitation of gestures, as in this situation the intended target position is demonstrated by the examiner and the patient has only to copy the movement. The case against a conceptual source of errors in the imitation of movements seems to be even more convincing if imitation is probed for meaningless and novel movements the execution of which cannot be helped by evocation of conceptual knowledge. Errors in the imitation of movements have therefore been a main argument for considering apraxia or at least a variety of it - as a disorder of movement execution [1,14-16,19]. The occurrence of such a disorder 
in the non-paralyzed left limbs seems to indicate a general dominance of the left-hemisphere for motor control $[14,16]$.

\section{Kinematics of apractic movements}

The hypothesis that apraxia is a manifestation of the left hemisphere's dominance for motor control has been further specified. It has been hypothesized that there is a left hemisphere dominance for the internal preprogramming of skilled movements of either hand [10,14]. By contrast, apraxia is said not to affect the guidance of movements by external feedback-control. Movements that evoke apraxic errors should differ from those that do not in that they pose heavier demands on internal preprogramming of movements and in that they are performed in the absence of external reference points which would allow feedback-controlled correction of errors.

The proportions of preprogrammed and feedback-controlled components of movements can be inferred from kinematic velocity profiles [13]. The preprogrammed phase of a target-directed movement is characterized by a 'bellshaped' velocity curve with one single velocity maximum [18]. Feedback controlled movement phases have multiple velocity peaks resulting from repeated updating and corrections of the movement path. The maximum velocity of the preprogrammed phase is generally higher than the velocity of the feedback-controlled phase.

Poizner et al. [20] analyzed the kinematics of pantomime of object use in apractic patients. They did not explicitly address the distinction between preprogrammed and feedback-controlled phases of movements but found multiple abnormalities of the kinematic profile, some of which (e.g. a reduction of maximum velocity) would be compatible with a specific deficit in the preprogramming of goal directed movements. However, a preponderance of hesitating, feedback-controlled movements in some apraxic patients does not by itself prove that the kinematic abnormalities are the source of apractic errors. Alternatively, if the patients lacked a clear conceptual representation of the intended target positions, they might have switched to a strategy of slow and feedback-controlled movements in an attempt to find the correct target positions by trial and error.

If lack of internal preprogramming is indeed the cause of apractic errors, it should be detectable in every movement which ends up with an apractic error. To test this prediction we undertook an investigation of movement kinematics in a larger sample of apractic patients [11]. We decided to investigate the imitation of meaningless gestures as errors in this task have been assumed to permit a direct observation of the executional deficit in apraxia $[1,4,14]$.

Arm and hand movements during the imitation of meaningless gestures were recorded using an ultrasonic device (CMS50, Zebris, Germany). Two tiny ultrasonic transmit- ters were fixed at the ulnar aspect of the wrist and of the metacarpophalangeal joint of the little finger on one hand and their positions in three-dimensional space were registered with a sampling rate of $100 \mathrm{~Hz}$. Calculation of movement durations, of path length, of velocities and of accelerations were done with the 3DA software [17].

Patients were asked to imitate simple hand postures demonstrated by the examiner. After five practice items, the trajectories of six movements were registered three times. For each movement it was denoted whether it ended in a correct imitation or not. For each correct imitation 1 point was credited. Neither any normal control nor any patient with right brain damage scored lower than 20 out of a maximum score of 23. Patients who fell below this score were classified as apractic.

In the velocity profiles of the wrist movement two phases could be distinguished: a transport phase during which the hand was brought very close to the target at the subject's head, and an adjustment phase during which the hand slowly approached its final position. For statistical evaluation, two parameters were considered: The number of velocity peaks during the transport phase, and the length of the distance travelled during the adjustment phase. In preprogrammed movements the transport phase should have only one velocity maximum and the adjustment phase should be very short if not entirely absent.

In 10 controls the left hand was examined and in 10 the right hand, but there were no difference between them. In the vast majority of movements $(97.9 \%)$ the transport phase yielded only one velocity peak. The hand was transported close to its final position with one single cycle of acceleration and deceleration. With very few exceptions the hand moved not at all or less than 30 millimeters after the termination of the transport phase, indicating that none or only minor adjustments were necessary to reach the final position. Apparently, imitation of hand postures is a very simple task for normal persons. Although the meaningless gestures are certainly unfamiliar, their kinematic profile discloses the features of a preprogrammed routine movement.

We examined 8 patients with right brain damage, and 20 patients with left brain damage and aphasia. Out of the aphasic patients, 13 were classified as apractic. All patients imitated the gestures with the hand ipsilateral to the lesion. The movement trajectories of right brain damaged patients did not markedly differ from those of the normal controls. By contrast, non-fluent and hesitating movements with multiple velocity peaks and prolonged adjustment paths were prevalent in patients with apraxia. There were, however, dissociations between the frequency of kinematic abnormalities and the diagnosis of apraxia. On the one hand, there were left brain damaged patients who succeeded in correctly imitating most of the positions but in whom the kinematic analysis revealed multiple velocity peaks or prolongations of the adjustment phase. Probably, these were patients with mild or recovered apraxia. On the 
other hand, and more importantly, there were two severely apractic patients in whom the majority of movements showed a completely normal kinematic profile. It may be of interest that these were the only apractic patients in whom the lesion extended into prefrontal cortex.

Analysis of the relationships between the kinematic profile and the end-position of single gestures revealed that the dissociation between a normal kinematic profile and an incorrect end-position was not restricted to these two patients but occurred in several trials of 2 additional apractic patients, being thus present in a significant num- ber of apractic patients. On the other hand, kinematically abnormal movements which eventually lead to the correct target position were a frequent finding in both, apractic and non-apractic left brain damaged patients but occurred only very rarely in controls or right brain damaged patients (Fig. 1).

The crucial finding of this study is that movements performed with the kinematic characteristics of largely preprogrammed movements can lead to apractic errors. This contradicts the assumption that kinematic abnormalities are the source of apractic errors. We think that a more

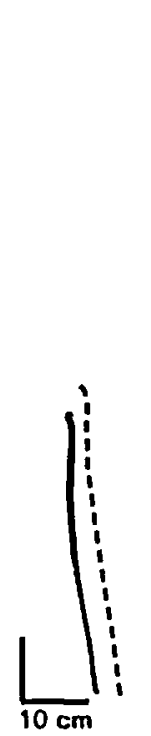

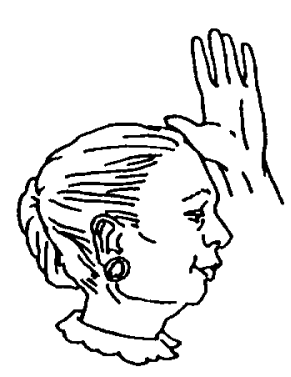
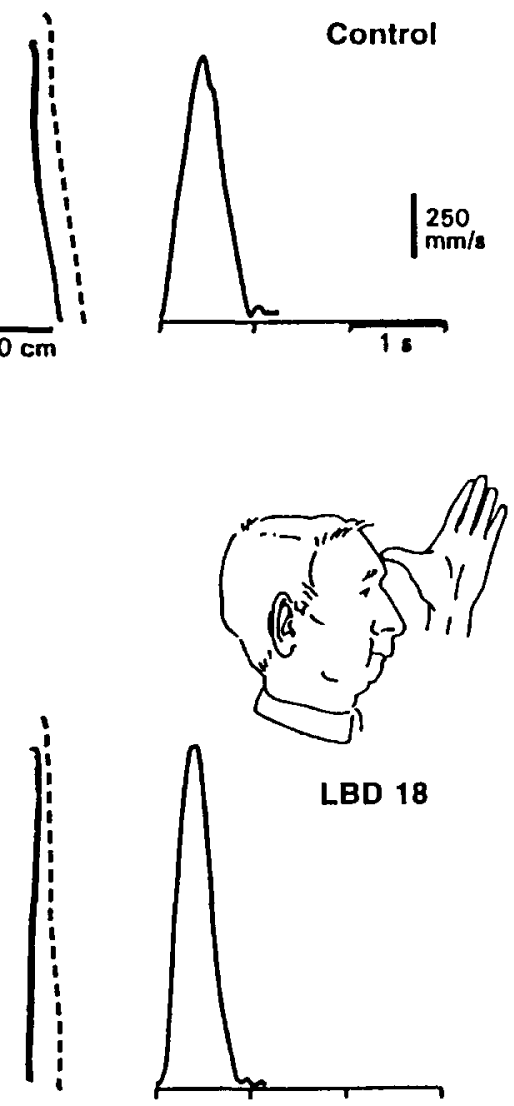

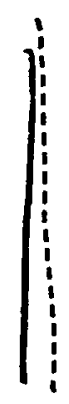
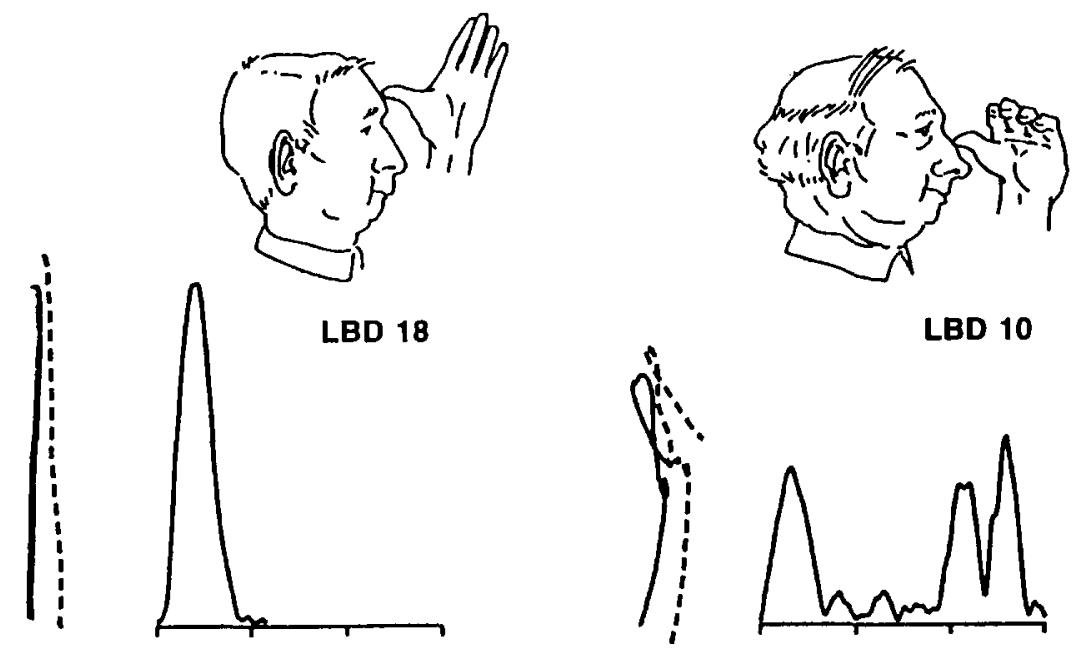

LBD 10

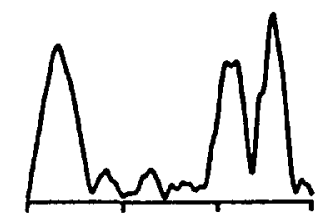

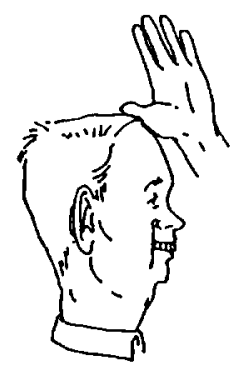

LBD 8b
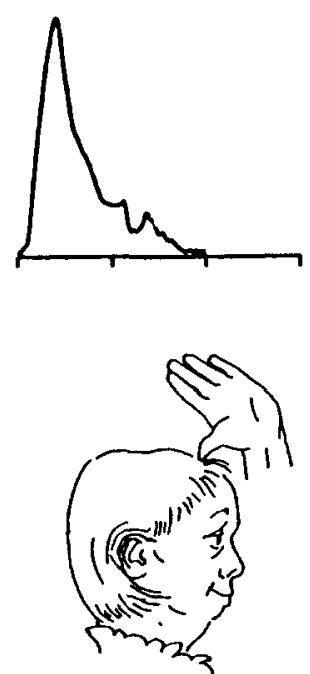

LBD 12

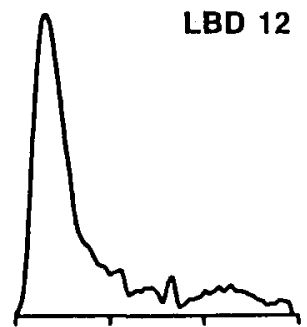

Fig. 1. Relationship between movement trajectories and final position in imitation of gestures. The sketch on the top of each graph shows the final position. The curves on the left side of each graph are the movement paths of the two markers attached to the ulnar side of the hand, the curve on the right shows the velocity profile of the proximal marker. In the control the target position is correct. The velocity profile consists of a single peaked transport phase and a very short final adjustment phase. LBD8a and LBD8b are two movements of a left hemisphere damaged patients who eventually reached the correct position too. However, velocity profiles reveal enhanced reliance on feedback-control: In LBD8a the transport phase has two velocity peaks and the adjustment phase is prolonged, in LBD8b the transport phase is single peaked but deceleration is irregular and the adjustment phase prolonged. LBD10 and LBD12 commit apractic errors and show kinematic evidence of lack of preprogramming of movement. By contrast, LBD18 reaches a wrong target position with a kinematically perfect movement. 
plausible explanation of the relationship between kinematic abnormalities and apractic errors could start from the assumption that apractic patients are unable to determine the exact target position of the intended movements. Whether or not insecurity about the target position leads to kinematic abnormalities depends on a choice of strategies. The patient may feel the difficulty of reaching a correct position and opt for a strategy of hesitant, feedback controlled movement, trying to find out the correct target during the course of the movement. Apparently this strategy was successful in a considerable number of trials and lead to dissociations between correct end-positions and abnormal kinematics. Alternatively, the patient may not care about the exact definition of the target position and may plan and execute appropriately a movement to an approximate final position. If the approximate position happens to be different from the required one, there results a dissociation between abnormal final position and normal kinematics. In the two patients in whom this was the prevailing type of apraxic errors, frontal lobe damage may have contributed to the lack of awareness of errors. In any case, the basic disturbance concerns the determination of the target position rather than the execution of the movement.

\section{Apraxia and the body scheme}

An idea which could account for deficient imitation of meaningless gestures without postulating a general motor dominance of the left hemisphere is, that the basic deficit in ideomotor apraxia concerns the ability to code and comprehend movements in relation to one's own body $[3,19,23]$. Both symbolic and meaningless gestures are aimed at producing explicitly defined positions or movements of body parts. By contrast, most movements outside the testing situation are aimed at and determined by external targets and are therefore spared from apraxia. The terms 'dyssomatognosie spatiale' [3,19] and 'reflexive apraxia' [23] have been proposed to characterize this conception of apraxia. It cannot account for faulty object and tool-use which consequently are assumed to be an expression of a basically different variant of apraxia. Frequently, the terms 'ideomotor' and 'ideational' apraxia are used to distinguish faulty performance of body-related movements from faulty object use [4,19].

The idea that the basic disturbance in ideomotor apraxia concerns the coding of movement in relation to one's own body is, however, open to several interpretations. There are three levels at which the 'body scheme' could be disturbed.

The disturbance could concern the implicit evaluation of the position and configuration of the body. The need to evaluate the position and configuration of one's body is implicit in the planning of any goal directed motor action $[2,13]$. Body parts are moved relative to other body parts, and external targets could not be reached if the initial position of the body and its parts were not taken into account. A disturbance of the implicit calculation of the position and configuration of one's body would manifest itself in misreaching and insecurity of movements to external objects even more than in the performance of movements in body centered space.

Alternatively, there could be a lack of explicit awareness of the own body's position and configuration. Explicit awareness of the position and configuration of the own body is rarely called on when movements are directed to external objects. It is, however, essential if the aim of the movement is to achieve a defined position or configuration of body parts.

Finally, the disorder could concern a general scheme of the human body which applies irrespectively of whether one own's body is concerned or not. Transposition of perceived target positions into a general concept of the human body may play a role in imitating gestures. Imitation of gestures has been said to test the integrity of a direct route from visual perception to motor control $[21,22]$, but on closer scrutiny it involves intermediate steps of considerable complexity. If the examiner sits opposite to the patient, the required movement is a mirror image of the perceived one. If, for another example, the examiner is taller than the patient, exact reproduction of their movement would overshoot the target on the patient's body. When gestures are to be imitated the target positions have to be inferred from a mental transposition of the demonstrated movement to the own body, and this transposition has to abstract from accidental determinants of the perceived movements as are, for example, the size or position of the demonstrating person. A feasible way to achieve this translation would be to conceptualize the perceived movements in their relationship to a general concept of the human body which applies irrespectively of the size and position of the body and irrespectively of whether the body belongs to the examiner, the patient, or anybody else.

\section{Manipulating a mannikin}

If defective imitation of meaningless gestures is caused by a lack of awareness of the position and configuration of only the patients' own bodies, they should be able to replicate the same gestures on an external model of the human body. If, by contrast, the basic disorder concerns a general concept of the human body, replication of the gestures on an external model should be as defective as on themselves. In order to distinguish between these possibilities imitation of meaningless gestures was examined in two conditions: on the patient's own body and on a mannikin [7].

The patients were asked to imitate 10 meaningless postures of the hand. The examiner set in front of the patient and demonstrated the posture 'like a mirror', that 
is, if the patients used their left hand, the examiner demonstrated with the right hand and vice versa. For each posture, 2 points were credited when imitation was correct after the first presentation. Otherwise, the demonstration was repeated and 1 point was given for a correct imitation on second trial. In addition to this conventional test of imitation, patients were asked to imitate the same gestures on a mannikin. They were seated opposite to a life-sized wooden mannikin whose arms and hands could be moved like that of a human being. They were asked to replicate with one hand of the mannikin postures demonstrated by the examiner. The examiner sat besides the mannikin and demonstrated the postures 'like the mannikin', that is, if the patient manipulated the mannikin's left hand, the examiner demonstrated with the left hand and vice versa. The postures used, the course of examination and the criteria for scoring were the same as with the imitation on the own body.

To rule out the possibility that a general deficiency of motor execution affects any skilled motor performance, a test of motor skill which required the manipulation of beads was included in the experimental design. It was hypothesized that a disorder of motor execution which manifests itself in tasks posing motor demands as different as imitation of gestures and manipulation of a mannikin would also show up in another test of manual dexterity. Patients were asked to take with one hand three beads from three vertical rods and to stick them on three other rods. They were not allowed to collect and transpose the beads one after the another, but had to collect all three beads in the hand before beginning to stick them on the target rods. The rods were aligned in a frontal plane. Patients who used their left hand moved the beads from the left set of three sticks to the right one, and vice versa. After two successful practice trials, 10 trials were run, and the time from leaving the starting point until the delivery of the last bead was measured with a stopwatch. If a bead fell out of the hand, the trial was repeated. The mean time of the 10 trials was taken for the statistical evaluation.

To explore whether a faulty apprehension of spatial relationships between body parts might be an expression of a more general disturbance of visuospatial processing, the comprehension and manipulation of spatial relationships outside the human body was assessed by the WAIS-R subtest block-design. As block-design demands motor manipulation of blocks, this test served as a further control for a general disturbance of motor execution. Finally, all patients with left hemisphere damage were administered the German version of the Token Test [12].

85 right-handed subjects were examined. There were 35 patients with left brain damage (LBD), 20 patients with right brain damage (RBD), and 30 controls without any evidence for brain damage. All patients with brain damage had suffered a single, unilateral cerebrovascular accident (CVA), and all LBD patients had aphasia. Patients with brain damage used the hand ipsilateral to the lesion for imitation of gestures, pantomime of object use and manipulation of beads and moved the same hand of the mannikin. Controls used either the right or the left hand for all tests. As there were no differences between controls using their right and those using their left hands the control data were collapsed.
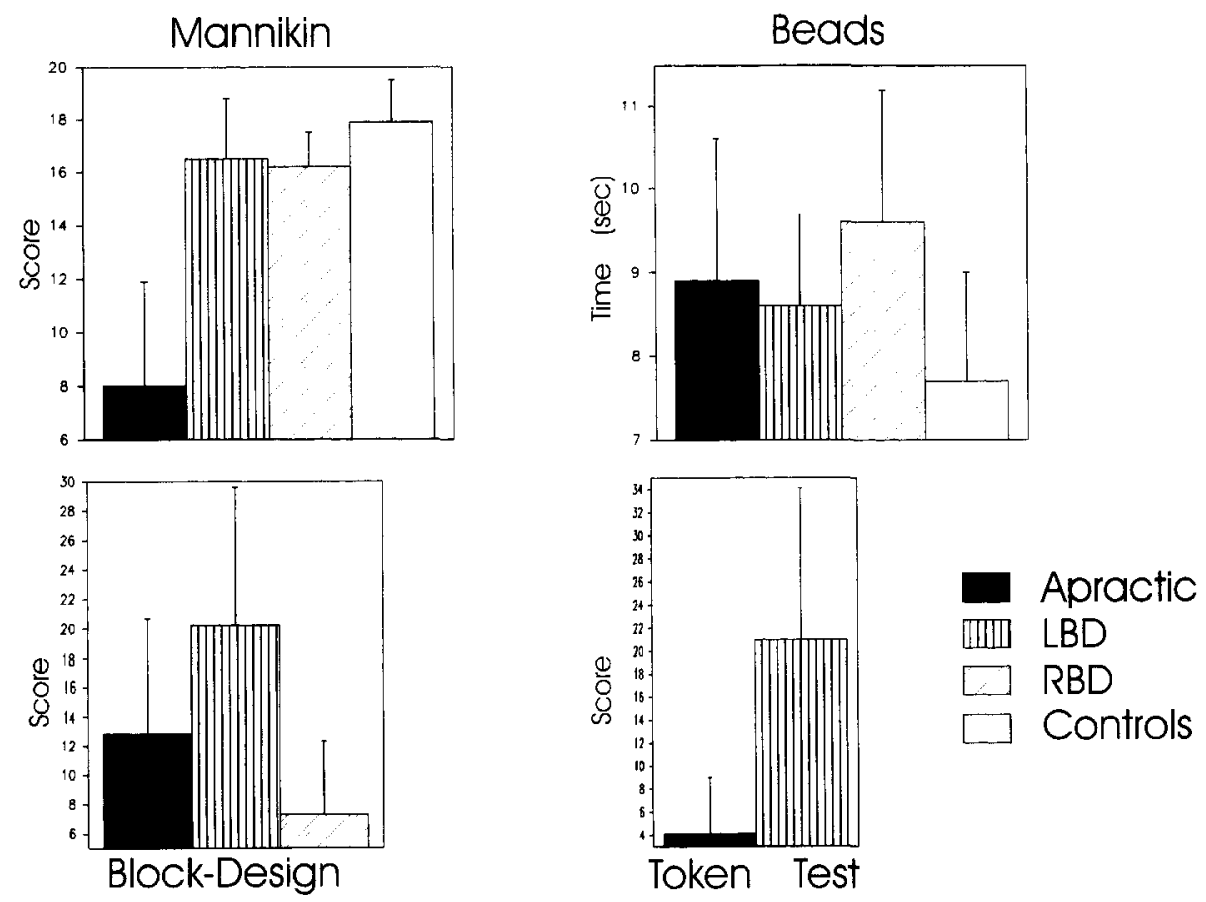

Fig. 2. Results of imitation on the mannikin, manipulation of beads, block design and Token Test. Means and S.D. are shown. See text for interpretation of results. 
Fig. 2 displays the main results. Apractic patients made significantly more errors than any other group when imitation was probed on the mannikin. Compared to left brain damaged patients without apraxia, they did also worse with Block-Design and were somewhat - albeit not significantly - slower with the manipulation of beads. However, on both of these tests, patients with right brain damage had more difficulties than the apractic patients. In manipulation of beads the right brain damaged patients' difficulties appeared to stem from the visuospatial demands of aligning the borings of the beads exactly with the direction of the rods. In sum it would appear that the difficulties which the apractic patients experienced with the mannikin can neither be referred to a general weakness of visuospatial abilities nor to a general lack of motor skill. The severity of aphasia was greater in apractic patients than in left brain damaged patients without apraxia and, consequently, the apractic patients' scores on the Token-Test were significantly lower. However, all patients had understood the instruction and imitation of gestures is itself a completely non-verbal task.

It is, in principle, impossible to ultimately prove that the associated occurrence of two neuropsychological symptoms stems from a common basic deficit, but the results of the study are clearly in accord with hypothesis that patients with apraxia have problems with a general concept of the human body, and that errors in the imitation of meaningless movement have their source there. This interpretation is far away from the initial assumption that impaired imitation of movements testifies the existence of a deficit of motor execution and hence a left hemisphere dominance for motor control. On the contrary, impaired imitation of movements can be seen as a further manifestation of non-verbal conceptual disturbances following left hemisphere damage $[5,8,9,24]$.

\section{References}

[1] Barbieri, C. and de Renzi, E., The executive and ideational components of apraxia, Cortex, 24 (1988) 535-544.

[2] Bizzi, E. and Mussa-Ivaldi, F.A., Motor control. In F. Boller and J. Grafman (Eds.), Handbook of Neuropsychology, Vol. 2, Elsevier, Amsterdam, 1990, pp. 229-244.

[3] de Ajuriaguerra, J., Hecaen, H. and Angelergues, R., Les apraxies. Varietes cliniques et lateralisation lesionelle, Rev. Neurol., 102 (1960) 566-591.
[4] de Renzi, E., Apraxia. In F. Boller and J. Grafman (Eds.), Handbook of Clinical Neuropsychology, Vol. 2, Elsevier, Amsterdam, 1990 , pp. 245-263.

[5] Finkelnburg, F.C., Sitzung der Niederrheinischen Gesellschaft in Bonn. Medizinische Section, Berl. Klin. Wochenschr., 7 (1870) $449-450,460-462$.

[6] Geschwind, N. and Damasio, A.R., Apraxia. In J.A.M. Frederiks (Ed.), Handbook of Clinical Neurology, Vol. 1 (49): Clinical Neuropsychology, Elsevier, Amsterdam, 1985, pp. 423-432.

[7] Goldenberg, G., Imitating gestures and manipulating a mannikin the representation of the human body in ideomotor apraxia, Neuropsychologia, 33 (1995) 63-72.

[8] Goldenberg, G., Aphasic patients' knowledge about the visual appearance of objects, Aphasiology, 9 (1995) 50-56.

[9] Goldenberg, G. and Artner, C., Visual imagery and knowledge about the visual appearance of objects in patients with posterior cerebral artery lesions, Brain Cogn., 15 (1991) 160-186.

[10] Haaland, K.Y., Harrington, D.L. and Yeo, R., The effects of task complexity on motor performance in left and right CVA patients, Neuropsychologia, 25 (1987) 783-794.

[11] Hermsdörfer, J., Mai, N., Spatt, J., Marquardt, C., Veltkamp and Goldenberg, G., Kinematic analysis of movement imitation in apraxia, submitted.

[12] Huber, W., Poeck, K., Weniger, D. and Willmes, K., Aachener Aphasie Test, Goettingen, Hogreve, 1983.

[13] Jeannerod, M., The neural and behavioural organization of goal-directed movements, Clarendon Press, Oxford 1988.

[14] Kimura, D., Neuromotor mechanisms in human communication, Oxford University Press-Clarendon Press, New York, 1993.

[15] Kimura, D. and Archibald, Y., Motor functions of the left hemisphere, Brain, 97 (1974) 337-350.

[16] Liepmann, H., Drei Aufsätze aus dem Apraxiegebiet, Karger, Berlin, 1908.

[17] Marquardt, C., Hermsdörfer, J. and Mai, N., 3DA - three-dimensional motion analysis, München, 1994.

[18] Morasso, P., Spatial control of hand movements, Exp. Brain Res., 42 (1981) 223-227.

[19] Morlaas, J., Contribution à l'étude de l'apraxie, Amàdéc Legrand, Paris, 1928.

[20] Poizner, H., Mack, L., Verfaellie, M., Rothi, L.J.G. and Heilman, K.M., Three-dimensional computergraphic analysis of apraxia, Brain, 113 (1990) 85-101.

[21] Rothi, L.J.G., Ochipa, C. and Heilman, K.M., A cognitive neuropsychological model of limb praxis, Cogn. Neuropsychol., 8 (1991) 443-458.

[22] Roy, E.A. and Hall, C., Limb apraxia: a process approach. In L. Proteau and D. Elliott (Eds.), Vision and Motor Control, Elsevier, Amsterdam, 1992, pp. 261-282.

[23] Schilder, P., The image and appearance of the human body, Kegan Paul, London, 1935.

[24] Vignolo, L.A., Non-verbal conceptual impairment in aphasia. In F. Boller and J. Grafman (Eds.), Handbook of Clinical Neuropsychology, Elsevier, Amsterdam, 1990, pp. 185-206. 